\title{
Methicillin-resistant Staphylococcus aureus (MRSA) Skin Infection after Epidural Anaesthesia - A case report -
}

CENTRO HOSPITALAR

LISBOA NORTE, EPE

Caldeira A., Albuquerque D., Henriques D., Monteiro M. Department of Anaesthesiology

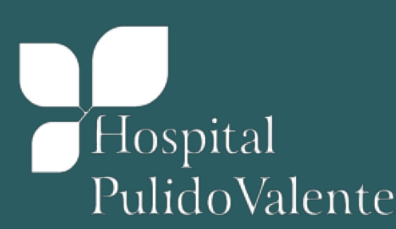

\section{BACKGROUND}

Regional anaesthetic (RA) techniques provide excellent anaesthesia and analgesia for surgical procedures, with many benefits compared to general anaesthesia. Most complications of RA are minor, but sometimes serious damage can occur like direct nerve damage, infection and spinal cord compromise.

Infectious complications may result in devastating morbidity, including abscess formation, necrotizing fasciitis, meningitis, paralysis and death.

\section{CASE REPORT:}

We report a case of MRSA skin infection after epidural anaesthesia in a 66-year-old man who was submitted to total knee arthroplasty under sequential combined-spinal epidural anaesthesia.

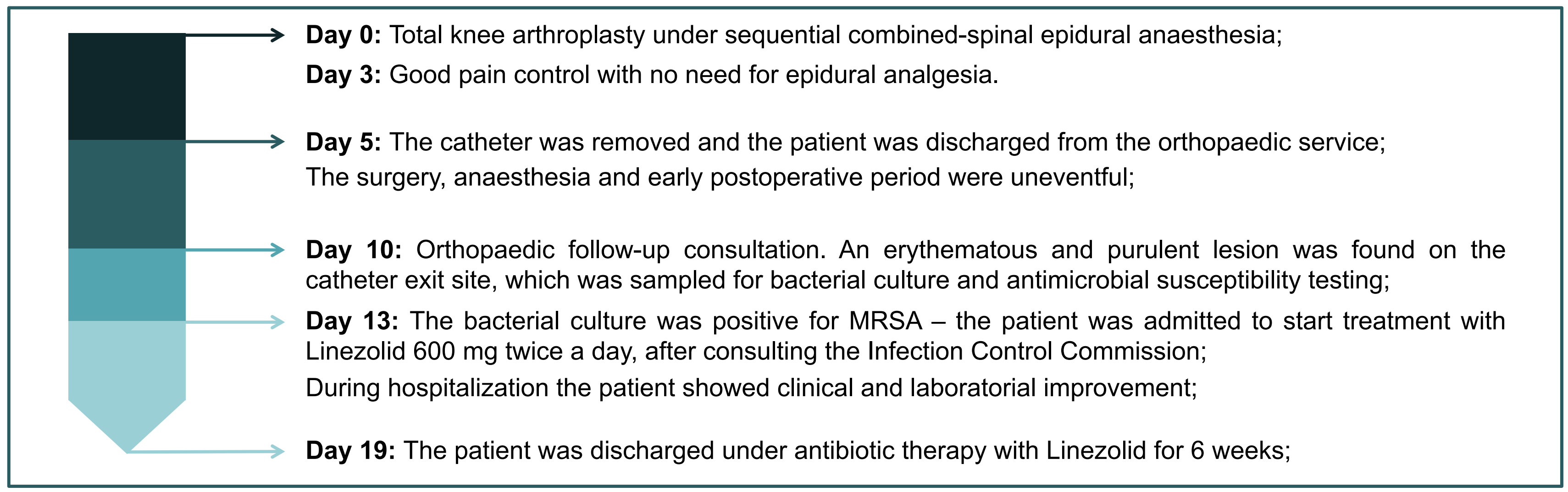

\section{DISCUSSION:}

Catheters should not remain in use longer than clinically necessary and, in our case, it could have been removed sooner as the patient's pain was already well controlled.

The use of bacterial filters may be considered during extended continuous epidural infusion, as in our case.

Aseptic technique and sterile occlusive dressings should always be used during the placement of neuraxial catheters, combined with a skin preparation such as clorhexidine.

Disconnection/reconnection of systems should be limited, to minimize the risk of complications.

\section{LEARNING POINTS}

Daily inspection and prompt removal of catheter should be done on any suspicion of infection or if no longer clinically necessary, as even healthy patients can develop infection, like the patient we are reporting in our case.

\section{REFERENCES:}

1. Novak-Jankovic, V., Infectious complications of regional anaesthesia and analgesia. Periodicum Biologorum. VOL.113, No 2,247-250,2011

2. Practice Advisory for the Prevention, Diagnosis and Management of Infectious Complications Associated with Neuraxial Techniques. Anesthesiology 2010;112:000-000 